\title{
tic\&société
}

Vol. $4, n^{\circ} 1 \mid 2010$

Interactivité et lien social

\section{Dans quelle mesure la TV sur mobile est-elle interactive?}

\section{Catherine Lejealle}

\section{(2) OpenEdition \\ Journals}

Édition électronique

URL : http://journals.openedition.org/ticetsociete/780

DOI : 10.4000/ticetsociete. 780

Éditeur

Association ARTIC

\section{Référence électronique}

Catherine Lejealle, "Dans quelle mesure la TV sur mobile est-elle interactive ? », tic\&société [En ligne], Vol. 4, n 1 | 2010, mis en ligne le 17 mai 2010, consulté le 02 mai 2019. URL : http:// journals.openedition.org/ticetsociete/780; DOI : 10.4000/ticetsociete.780 


\title{
Dans quelle mesure la TV sur mobile est-elle interactive?
}

\author{
Catherine Lejealle \\ Direction de la Recherche Telecom Paris Tech \\ Département Sciences Economiques et Sociales (SES) \\ 46 rue Barrault \\ F - 75013 Paris \\ Tel.: ++33 (0)619474387 ou ++33 (0)147599893 \\ catherine.lejealle@gmail.com
}

\begin{abstract}
Ingénieur télécom et docteur en sociologie, chercheur à Télécom Paris Tech au Département Sciences Economiques et Sociales, Catherine Lejealle s'intéresse aux processus d'appropriation des TIC par les utilisateurs et à la mobilisation des objets dans la construction de soi et de son rapport aux autres : jeux, télévision mobile, sites de rencontres, usages professionnels du mobile chez les cadres français et californiens. Elle est l'auteur de Les usages du jeu sur le téléphone portable : une mobilisation dynamique des formes de sociabilité (L'Harmattant) et de La télévision mobile : usages, contenus et nomadisme (L'Harmattan).
\end{abstract}




\title{
Dans quelle mesure la TV sur mobile est-elle interactive?
}

\section{Résumé}

L'offre actuelle de TV mobile est peu interactive car il n'y a pas voie retour permettant un contrôle sur le contenu diffusé en temps réel. Néanmoins, cette faible interactivité ne frustre pas parce que la motivation d'usage consiste moins à regarder la TV qu'à partager un moment ou à s'abriter derrière l'écran pour s'isoler au sein du collectif. Au travail, au domicile ou en mobilité, on joue avec sa présence et son absence. L'usage permet de s'approprier l'espace différemment en s'absorbant ponctuellement dans le visionnage tout en n'oubliant jamais le contexte extérieur et la vigilance qu'il demande.

Mots-clés : Mobilité, interactivité, télévision, téléphone portable

\begin{abstract}
Current mobile TV has limited interactivity: it's a one-way connection with no ability to influence the content and limited choice of timing. In spite of that, this low level of interactivity is not frustrating as the purpose is less to watch TV than to either to share a moment with colleagues and friends or to hide behind a screen to be alone in a crowded environment. Mobile TV gives people an opportunity either to be with others or to be alone, depending on their mood. At work, at home or on the move, people can play at being present or absent. The use of the mobile phone enables a different appropriation of space by punctual involvement with one's mobile screen, all the while giving the external environment all the attention it requires.
\end{abstract}

Keywords: Interactivity, mobility, mobile phone, television

\section{Resumen}

La oferta actual de TV móvil es poco interactiva debido a que no hay vía de retorno que permita un control sobre el contenido difundido en tiempo real. Sin embargo, esta débil interactividad no es frustante porque la motivación del uso consiste menos en observar la TV que en compartir el momento o en refugiarse detrás de la pantalla para aislarse en un medio colectivo. En el trabajo, en el domicilio o en movilidad, se juega con su presencia y su ausencia. El uso permite apropiarse del espacio de manera diferente, ya que 
Dans quelle mesure la TV sur mobile est-elle interactive?

es puntualmente absorbido en el visionado, sin que eso signifique olvidar el contexto exterior y el estar alerta que dicho uso supone.

Palabras claves : Interactividad, movilidad, teléfono móvil, televisión 
Depuis un ou deux ans, les chaînes de télévision enrichissent leur offre en proposant de voir en différé ou de revoir certaines de leurs émissions. Ce visionnage se fait sur ordinateur en passant par leur site. Cette offre dite Replay ou Catch up TV permet une délinéarisation des usages, c'est-à-dire un affranchissement par rapport à la grille horaire. Une fois diffusés, on peut regarder ces programmes n'importe quand. La contrainte temporelle saute. Le téléspectateur peut regarder un programme à l'heure qu'il souhaite et selon des modalités flexibles. Par ailleurs, des chaînes dites webtv exclusivement accessibles depuis Internet ont vu le jour, permettant à l'usager de regarder à l'heure qui lui convient en se créant son propre programme en fonction de ses goûts mais dans la limite souvent étroite des offres actuelles. Enfin, une étape supplémentaire est franchie, avec la promesse des opérateurs de téléphonie mobile de se libérer de la contrainte spatiale. Dès lors que l'on dispose d'un mobile 3G, il est désormais possible de regarder la TV n'importe où et n'importe quand. Des bouquets de quelques cinquante chaînes sont offerts pour sept à dix euros mensuels en usage illimité sur un mobile. Ce sont des programmes de flux car dépendant de l'heure de diffusion. S'y s'ajoutent quelques programmes de stock en mode replay, c'est-à-dire des archives de programmes déjà diffusés. Sur le plan technique, le service TV mobile fonctionne sur le réseau $3 \mathrm{G}$ existant depuis plusieurs années ${ }^{1}$, ce qui suppose des contraintes en terme de qualité de service. Ce service promet une interactivité associées aux deux libertés, tant temporelle que spatiale. Dans quelle mesure peut-on parler d'interactivité à propos de la TV mobile? Quelles sont les attentes des usagers?

Après avoir rappelé quelques fondamentaux de la notion d'interactivité, nous décrivons son application à la télévision que nous illustrons à l'aide d'une enquête réalisée sur des usagers de la TV mobile sur la base d'entretiens qualitatifs en face-à-face. Nous avons procédé en trois étapes : deux entretiens en face-à-face distants de quinze jours, pendant lesquels l'enquêté tient un carnet de bord. Rempli à chaque usage de la TV mobile, il comprend date de visionnage, heure de début et heure de fin, lieu, activité précédente et activité suivante, interruptions éventuelles et conséquence sur le visionnage (arrêt, changement de chaîne...), tiers présents avec la nature de leur participation (ils regardent avec, ils travaillent à côté, ils dorment...), choix du ou des programmes. Le premier entretien aborde les contraintes d'emploi du temps, les

1 Dans quelques temps, cette TV mobile sur réseau $3 G$ sera remplacée par la télévision mobile personnelle (TMP). La TMP repose sur la norme DVB-H (Digital Video Broadcasting Handhelds) voisine de la TNT terrestre mais qui demande de déployer de nouvelles infrastructures.

tic\&société - 4 (1), 2010 
modes de transport utilisés, les usages généraux du mobile (appels, SMS, MMS, choix de sonneries, choix de fond d'écran, photographies, vidéo, visioconférence, musique, surf sur Internet...), l'articulation avec les autres pratiques dont la TV classique et l'entrelacement avec les appels téléphoniques et les SMS. Au cours du second entretien, l'usager doit commenter chaque ligne de son carnet de bord.

Le choix de la population des répondants a tenu compte des travaux existant actuellement sur la TV mobile (Sodergard, 2003 ; Goggin, 2007 ; O'Hara, Slayden, Vorbau, 2007). Or, les répondants choisis sont essentiellement des cadres supérieurs, des consommateurs précoces de la TV mobile (early adopters) ou des technophiles avertis. Les études INSEE conduites par Olivier Donnat (2009) montrent pourtant que les plus gros consommateurs de TV domestique sont les catégories socioprofessionnelles inférieures (CSP). Dès lors, il nous a semblé plus pertinent de nous concentrer sur ces CSP inférieures et moyennes inférieures.

Nous avons interrogé quarante Franciliens, en équipartition homme / femme et appartenant aux CSP inférieures ou moyennes inférieures (femme de ménage, publiposteur, secrétaire, animateur de têtes de gondole en hypermarchés, " homme à tout faire dans un hôtel »...). Agés de vingt et un à quarante cinq ans, ils ont tous souscrit à l'option TV mobile.

Le recrutement a été confié à une société spécialisée, garantissant une totale indépendance des usagers entre eux et vis-à-vis de l'enquêteur. On a cherché à décrire une grande diversité de situations pouvant avoir un impact sur les pratiques : statut familial (marié ou en couple, avec ou sans enfants...), travail sédentaire ou avec déplacements et enfin, nature des moyens de transport usuels. Nous avons fait varier ces paramètres car l'usage est lié aux lieux fréquentés et à l'emploi du temps. En effet, un usager venant d'acquérir un scooter huit jours auparavant constatait que les deux heures de visionnage de la TV sur mobile qui occupaient les heures de transports quotidiennes avaient disparu du fait du changement de motorisation.

\section{Définition de la notion d'interactivité}

\subsection{De la machine intelligente au dispositif}

Le terme interactivité s'est progressivement imposé dans la littérature comme dans les publicités pour des dispositifs ou dees services de communication au point de devenir un mot valise. Avant l'arrivée de dispositifs qui permettent la médiation via les TIC (dits Computer-Mediated-Communication 
ou $\mathrm{CMC}$ ) l'interactivité était liée au face-à-face entre deux personnes. Elle se confondait souvent avec l'interaction en coprésence.

Aujourd'hui, avec la pénétration des TIC dans toutes les sphères des activités humaines et le développement de la réalité virtuelle, ce terme recouvre une variété de situations. Les communications médiées entre tiers géographiquement distants mais aussi entre un tiers et une machine dite intelligente entrent dans le spectre de la définition. En effet, à la différence du réfrigérateur ou du four traditionnel, les machines intelligentes permettent le dialogue avec l'usager et offrent une vaste panoplie de modes opératoires et d'usages aussi complexes que variés. Ainsi, la télévision peut-elle servir de bruit de fond pour masquer le silence, de pédagogue qui diffuse un programme didactique, de garde d'enfants ou encore de support relationnel à la discussion. Elle peut divertir, informer, manipuler, éduquer, occuper ou encore servir de point d'appui à la discussion entre proches ou collègues. Les machines interactives sont des objets techniques sophistiqués qui peuvent dialoguer avec l'environnement et dont le programme peut évoluer en temps réel en fonction des modifications apportées par les usagers. A ce titre, la notion d'objet ellemême se transforme. Elle renvoie à un rapport utilitaire, fonctionnel et à une utilisation susceptible de déboucher sur une opération ou un résultat.

Certains auteurs préfèrent la notion de dispositif interactif considérant que le dispositif est plus complexe et tient compte d'une orientation vers les normes sociales. Un dispositif est conçu en intégrant les usagers ou les normes d'usages dès la création. II y a rétroaction du social sur le technique. D'autres encore, en dignes héritiers de Veblen (1970) et de Simmel (1908) insistent sur la consommation ostentatoire ou sur la différenciation sociale. La possession et l'usage plus ou moins ostentatoires de tels objets indiquent un statut social et participent à la construction identitaire. Enfin, d'autres auteurs comme Chambat (1994) ou Pronovost (1994) préfèrent la notion de "filière d'usage » et de " filière d'objets » pour ces usages dits «banalisés » de la vie quotidienne et qui véhiculent avec eux une certaine notion du rapport à l'objet. GarabuauMoussaoui et Desjeux (2000) analysent l'objet banal au quotidien, objet révélateur des relations sociales. L'objet peut donc être considéré comme un outil, un signe, un objet statutaire ou encore un dispositif.

La machine interactive présente encore une autre différence avec la machine traditionnelle. Pensée comme telle lors de la conception, la machine interactive fonctionne essentiellement en réponse à une stimulation humaine. A la différence du réfrigérateur passif qui fonctionne en continu sans intervention humaine, un ordinateur ou un mobile ne fonctionnent que si on s'en sert. La machine interactive est alors davantage active mais implique une relation 
d'action et de réaction. Ceci demande de définir au préalable des procédures langagières et de partager un code avec l'usager. Ce point pose d'ailleurs souvent problème car l'usager se trouve parfois perplexe devant la complexité technique d'objets dont les publicités lui vantent la facilité et le fonctionnement en langage naturel, en « touch and feel ».

Cette interaction entre la machine et l'usager conduit à un usage le plus souvent individuel, personnalisé, en tout cas loin du modèle des médias de masse et de la diffusion unilatérale comme c'est le cas de la télévision classique. L'usager entend la promesse de programmes à la carte, quand il le veut et comme il le veut, à l'image des playlists sur les lecteurs MP3 ou les radios en ligne.

\subsection{La notion d'interactivité}

Dans son Vocabulaire des techniques de l'information et de la communication (TIC), la Commission générale de terminologie et de néologie définit l'adjectif "interactif» comme ce qui "qualifie » les matériels, programmes, ou les conditions d'exploitation qui permettent des actions réciproques en mode dialogué avec des utilisateurs ou en temps réel avec des appareils " (Commission générale de terminologie et de néologie, 2009, p. 105). Cette même définition est publiée au Journal Officiel du 22 septembre 2000.

Sur le plan théorique, les chercheurs confirment que l'interactivité s'oppose à une communication où l'interlocuteur est passif. Hodge (1994) définit l'interactivité comme une communication dans les deux sens (locuteur/interlocuteur). Jensen (1999) dira que l'interactivité offre aux usagers la possibilité d'influencer le contenu ou/et la forme de la communication médiée. En parlant de possibilité, il reconnaît également que l'usager peut - au moins par moments - opter pour un rôle plus passif comme le spectateur de la télévision classique. Ainsi, un service interactif permet-il de prendre le contrôle sur ce qui est diffusé ou émis mais aussi sur le moment de la diffusion.

D'autres chercheurs tels que Feldman (1997) ou Steuer (1995) complètent cette définition en insistant sur le fait que l'usager participe activement à ce qui lui est transmis en pouvant modifier en temps réel à la fois la forme et le contenu de l'information. Enfin, appliqués aux médias de diffusion en temps réel, Downes et Mc Millan (2000) modélisent l'interactivité en distinguant six dimensions, dont trois sont liées aux messages et trois aux usagers à savoir :

- $\quad$ une communication à deux sens ; 
- un horaire de diffusion et de programmation ou d'émission flexible pour répondre à la demande des participants ;

- un environnement de communication qui crée une sensation de place comme c'est le cas pour Internet et les forums de discussion ;

- le contrôle de l'environnement par les participants ;

- la possibilité de personnaliser ce qui est communiqué ;

- le but de la communication n'est pas de convaincre ou de vendre. II est davantage orienté vers l'échange et l'égalité.

L'interactivité s'oppose donc à la diffusion unilatérale d'un émetteur vers $\mathrm{N}$ récepteurs, sans feedback ou rétro-action en temps réel.

\subsection{La TV interactive}

Déjà réalité dans certains pays, la TV interactive reste souvent limitée au domicile. Néanmoins, elle offre comme nouveauté un canal retour intégré au dispositif. Signalons que le retour est possible depuis longtemps, mais il nécessite un dispositif complémentaire et il est collectif. En effet, les spectateurs comme les auditeurs de radio ont la possibilité de voter en temps réel pour la suite du programme, avec des émissions du style " stop ou encore " pour la musique, ou pour éliminer un candidat (Star Académy, Loft Story). L'usager saisit son téléphone fixe ou mobile pour appeler ou envoyer un SMS et vote. L'impact individuel est différé et collectif. D'autres émissions, par exemple des programmes destinés aux enfants lui permettent de dessiner un pont ou une route pour sauver un héros. Le dessin est fait sur une feuille de papier que l'enfant devait coller à l'écran sans que l'émetteur n'ait prise sur le bon fonctionnement de la procédure. Si l'enfant ne comprend pas la manipulation ou s'y refuse, l'émission continue.

Ces innovations amènent à redéfinir le rôle de l'usager qui, comme le dit Christensen (2002), devient à la fois auditeur, spectateur, consommateur et producteur du contenu. Pour cela, Rutten et al. (2003) se proposent de repartir de la chaîne de valeur appliquée au secteur culturel alliant la création de contenu, sa production, son édition, sa distribution et sa consommation/son usage pour voir à chaque niveau si l'offre interactive permet l'implication de l'usager. Ils soulignent que l'offre actuelle se limite au dernier stade.

Van Dijk et al. (2003) distinguent quatre rôles d'interactivité pour l'usager : Il peut zapper, réagir à ce qui est proposé, agir en participant au contenu en cours de création et enfin, échanger. Mais leurs enquêtes montrent que les usagers 
veulent uniquement zapper, voter et surtout pouvoir maîtriser les horaires de diffusion. Ils acceptent éventuellement d'acheter ou de parier mais en aucun cas d'investir en amont ou en aval la chaîne de valeur. La TV appartient à l'univers des dispositifs dits "lean back», devant lequel l'usager est passif. Ces dispositifs "lean back» ont un unique canal de diffusion et permettent par construction très peu d'interactivité. Ils invitent à adopter une position couchée lors de l'usage. Au contraire, les dispositifs comme l'ordinateur dits «lean forward " présentent une voie de retour facilitant l'interaction avec les autres usagers ou avec le dispositif. L'usager a davantage tendance à adopter une posture face à l'écran dite « forward » et moins une position couchée.

\section{L'interactivité de l'usage de la TV mobile}

\subsection{L'interactivité de l'offre}

L'interactivité du service de TV mobile est aujourd'hui limitée car il n'y a pas de voie retour pour interagir avec la machine. L'usager n'a aucune maîtrise du contenu diffusé et seulement une faible maîtrise du temps puisque les programmes en stock sont encore rares. II ne peut ni voter, ni influer en direct sur le programme. La nouveauté consiste pour les opérateurs et fournisseurs de contenu à proposer en mode "push " des nouveautés de programme ou de chaînes qui viennent enrichir le bouquet. Ce fut le cas pour Secret Story. Avertis que l'émission démarrait, les usagers ont répondu positivement à cette invitation. Ils ont alors découvert des contenus spécifiques au mobile comme Les coulisses de Secret Story. Par ailleurs, certaines séries permettent une amorce de maîtrise du temps en proposant d'aller directement au second ou au dernier tiers de l'épisode pour éviter de revoir le début. Ces propositions sont acceptées car elles remplissent deux conditions nécessaires.

Primo, la télévision faisant l'objet d'un usage relationnel (on en parle avec les collègues et les proches à défaut de regarder ensemble), le programme doit passer durant la même période sur le mobile et sur le poste classique. II peut s'agir d'un programme ou d'un format spécifiques au mobile mais il doit être dérivé du programme nominal, comme ce fut le cas pour Les Coulisses ou pour des bonus et des making off. Autrement dit, si la série Colombo ne passe sur aucune chaîne, elle n'attirera pas le mobinaute.

Secundo, du fait du temps de visionnage disponible sur le mobile souvent réduit à des tranches de cinq à dix minutes, la durée du programme mis en push doit être compatible avec cette contrainte, soit parce qu'il dure ce temps-là, soit parce qu'il peut être fragmenté pour s'y adapter. Si ces deux conditions ne sont pas réunies, des refus sont constatés. Par rapport à la définition, l'interactivité 
est quasi nulle puisqu'elle ne permet qu'un faible contrôle et est davantage subie que choisie. Les usagers se plaignent notamment lorsque leur opérateur change l'ordre d'apparition des chaînes. Comme dans un hypermarché, l'usager aime conserver ses repères : " Je sais que ma chaîne est en page 3 sur 5 et j'y vais directement. Lorsqu'ils me les changent, ça me perturbe et me fait perdre du temps ».

\subsection{L'interactivité liée au combiné}

L'interactivité de l'offre actuelle est encore limitée. Elle s'apparente davantage à des interruptions et des ruptures qui cadencent et rythment le visionnage. Ceci n'empêche pas les usagers de considérer l'usage comme interactif parce que « Ça bouge... Ça n'est pas linéaire et prévisible comme la TV... II y a de l'animation... Ça change tout le temps ». A ce titre, elle est à la fois valorisée (moins d'heures passées passivement devant le poste) et redoutée car subie, imprévisible et non maîtrisable. L'usager n'a en effet aucune emprise sur les changements de présentation du service. Le nouveau paradigme du changement, de la flexibilité, de l'activité comme valeur positive joue pleinement car refuser la nouveauté fait passer pour "ringard et vieux». Entrer en dissonance avec les représentations sociales est source de souffrance pour eux si bien qu'ils « font avec plutôt que contre ».

D'autres interactions liées aux caractéristiques du mobile contribuent à cette impression de mouvement et d'activité qu'eux qualifie d'interactivité. En effet, transportable et nomade, il ouvre à de nouveaux contextes d'usages notamment en mobilité et au travail. De plus, la batterie, la mémoire, l'écran et le clavier étant limités, l'expérience de la TV n'est pas transposable sur le mobile. La TV mobile demande une vigilance permanente et des ajustements. Ainsi, en fin de journée, si la batterie ne suffit pas jusqu'au soir, l'usager cesse ou renonce à l'usage ludique, de peur de ne plus pouvoir appeler de son mobile en cas de problème. De même, pour laisser de la place en mémoire aux photographies ou à la musique, certains enquêtés préfèrent ne pas stocker de films jugés trop gourmands. Si ces arbitrages entre usages sont défavorables à la TV mobile, d'autres limitations peuvent se transformer en avantages: la petite taille de l'écran en fait un outil discret qui permet de regarder la TV dans les lieux où le mobile est interdit (milieu médical) ou ceux où il est parfois toléré mais où l'usage de la TV ne l'est pas (travail). Ce petit écran permet de regarder la TV à l'insu des collègues, du supérieur hiérarchique, du personnel médical ou d'inconnus coprésents. II permet de transgresser des normes d'usage pour le plus grand plaisir des usagers et parfois avec la complicité de collègues, créant une intimité supplémentaire. 
De plus, le support étant d'abord un outil de communication, l'appel entrant, le SMS ou le MMS sont prioritaires et interrompent le service sans que celui-ci ne revienne automatiquement en fin de communication. L'usager doit réinitialiser manuellement la connexion et redémarrer le service, en ayant perdu la tranche d'émission ou la partie en cours. II peut alors y renoncer ou, se rappelant qu'il a aussi un téléphone entre les mains, appeler d'autres personnes. L'interruption est alors source de distraction et de gêne qui peut inviter à passer à autre chose.

\subsection{L'interactivité liée au réseau de l'opérateur}

Une autre forme d'interactivité dérive de l'opérateur. Tributaire de la qualité de réception du réseau de l'opérateur, la TV mobile s'interrompt fréquemment dans les parties souterraines des transports en commun. Ceci en fait un service aléatoire, peu fiable, très différent « du presse-bouton qu'on a à la maison ». De plus, la luminosité évoluant lors des déplacements, l'usager doit sans cesse adapter l'inclinaison de l'écran pour maximiser la visibilité. II s'agit bien d'un téléphone sur lequel on a greffé un téléviseur mais en accordant la priorité à cette fonction initiale.

\section{L'interactivité liée aux autres personnes}

\subsection{Un usage principal, secondaire ou tertiaire}

L'usage sort du salon pour investir à la fois des lieux postés (travail) et des lieux de transit (file d'attente de magasins ou de cinémas), en mode immersif ou en superposition à une autre activité. Le contexte apporte de l'interactivité à travers la gestion des interruptions liées aux tiers présents (inconnus dans le métro, connus au domicile et au travail) et l'autre activité qui se superpose éventuellement (descendre du bus, répondre au conjoint, surveiller le repas ou son enfant). En effet, en reprenant la terminologie d'autres chercheurs (Van den Broeck et al., 2006), on observe trois situations d'usage, selon que la télévision est l'activité principale, secondaire ou tertiaire et que la télévision est regardée de face, d'à côté ou qu'elle est allumée en arrière plan. La nécessité de faire une pause ou de fragmenter les usages ainsi que le niveau d'attention et de disponibilité diffèrent d'une situation à l'autre. 


\subsection{Le choix du contenu}

On observe que le choix du contenu se fait en fonction de la situation. S'ils pensent avoir cinq à dix minutes, le temps d'arriver à leur station de métro ou le temps que la machine à café prépare leur boisson, ils optent pour un format court, facile à comprendre : un programme "pas prise de tête dans lequel je rentre tout de suite et que je peux abandonner sans regret ». Les contenus privilégiés sont alors les flashs d'information, la musique et le sport. S'ils doivent s'occuper pendant une durée longue (trajet en train), ils optent pour un programme long comme les reportages. Les usagers veillent à avoir en permanence une palette de cinq chaînes complémentaires pour s'adapter aux contraintes temporelles et spatiales.

Par conséquent, les contenus regardés sur la TV mobile sont essentiellement des jeux et divertissements, de la musique, du sport et des informations, contenus moins consultés sur le poste de télévision classique. Ces différences entre supports sont davantage liées au contexte qu'aux goûts des usagers en matière de programmes ou qu'à l'offre elle-même. Ces contenus ont la particularité de pouvoir être pris en cours de route et abandonnés en chemin. L'usager peut les fragmenter pour les adapter à une durée souvent inconnue à l'avance (attente d'un tiers ou d'un événement), un événement arrivant n'importe quand, ce qui n'est pas simple avec un film ou une série si bien qu'ils continuent à les regarder sur leurs téléviseurs au domicile. Ces contenus élastiques, à la demande, présentent l'autre avantage d'exiger une moindre attention qu'un film ou une série policière. Ils peuvent être mis en bruit de fond lorsque la situation exige une grande attention comme c'est le cas dans les endroits où l'usager a peur du vol ou lorsqu'il doit se partager entre plusieurs écrans (notamment dans le cadre professionnel).

\subsection{Un usage collectif ou individuel, récurrent ou non}

La motivation d'usage ne consiste pas tant à regarder qu'à disposer d'une ressource supplémentaire pour concilier collectif et individuel, pour être libre ensemble (Singly, 2001). En effet, au travail, au domicile et en mobilité, situations recoupant un tiers des usages de la TV mobile, on observe une oscillation entre plusieurs modalités d'usages, les unes collectives, les autres individuelles. Avec les collègues ou les proches, l'usage peut être collectif et récurrent (visionnage d'une émission favorite, souvent une série ou le journal télévisé). L'accès est direct et l'heure de diffusion structurante dans l'emploi du temps des usagers qui n'hésitent pas à avancer l'heure du déjeuner pour s'y caler. Le partage fait lien et crée une routine. "Comme on n'a pas la télé au 
Dans quelle mesure la TV sur mobile est-elle interactive?

bureau, mon portable me permet de regarder des feuilletons comme Les feux de l'amour avec mes collègues. A 13 heures 45, on l'allume pour ne pas rater le début et on va jusqu'à la fin. On déjeune plus tôt pour être prêtes à l'heure. Parfois on appelle une cousine qui regarde avec ses collègues. On commente. " L'usage collectif n'est pas nécessairement récurrent mais ponctuel car lié à une actualité du moment dite " chaude » telle que libération d'otages, résultats d'élections ou résultats sportifs... Les usagers interrompent parfois la tâche en cours pour consulter de manière réitérée la TV mobile afin d'obtenir une information ou son actualisation. Attendue par tout un service, cette information s'intègre dans la conversation et donne lieu à commentaires et à partage narratif. Elle crée du lien entre collègues sur le lieu de travail en incluant parfois le supérieur hiérarchique.

A côté de ces usages collectifs s'ajoutent les usages individuels. Ils peuvent concerner les salariés isolés (vendeur de magasin, conducteur de bus, commercial en clientèle) qui attendent de la TV mobile une compagnie et la laissent parfois des heures en bruit de fond sans continuellement regarder l'écran. Mais le plus souvent, ils concernent les salariés qui évoluent dans un environnement collectif jugé bruyant et parfois pesant. Ils tentent ainsi de s'isoler dans ce qu'ils qualifient « de bulle protectrice», le temps d'une pause. L'usage sert d'exutoire aux tensions si bien que le contenu importe peu et que l'interactivité proposée par le service est moins importante que celle liée au contexte. II s'agit davantage de zapper, d'évacuer le stress avec ce qu'Albert Memmi (1979) qualifie d'" objet d'apaisement moteur", qui pourrait être la cigarette, le chocolat ou le café. L'usager aspire à se mettre en retrait, à se soustraire au collectif, "à être là sans être là ». C'est une modalité d'usage relationnel particulier, au sens où il permet de rendre la présence des autres supportable, non par partage mais par isolement. La TV mobile apparaît comme une ressource supplémentaire pour faire face à l'injonction paradoxale et de plus en plus fréquente en entreprise, de devoir à la fois échanger - coopérer être transparent et être compétitif, performant. Cette concurrence est renforcée par tout un dispositif d'évaluation et de rémunération individualisée qui va à l'encontre de l'échange et du partage de bonnes pratiques. Par conséquent, l'entrelacement apparent entre pratiques télévisuelles (loisir) et activité salariée correspond en réalité à l'entrelacement de deux activités professionnelles car dans ce cas spécifique au travail, l'usage de la TV mobile n'est pas assimilé à une pratique de loisir (regarder la télévision) ou à un temps personnel gagné sur le temps de travail mais à une modalité de gestion de sa présence au travail. 


\section{Conclusion}

L'interactivité de la TV mobile est encore très faible car elle ne repose pas sur une voie de retour permettant un contrôle du contenu diffusé en temps réel. La maîtrise du temps n'est guère améliorée par rapport à la TV classique car les programmes de flux priment sur les programmes de stock. II s'agit davantage d'animation et de réactivité pour s'ajuster aux limitations du dispositif et aux évolutions du contexte (coupure réseau, luminosité changeante, ordre des chaînes modifié).

Néanmoins, peu d'usagers sont frustrés de cette faible interactivité parce qu'il s'agit moins pour eux de regarder la TV (vocable que les enquêtés utilisent rarement) que d'utiliser cet usage au service du lien social. L'usage n'est pas une activité séparée des autres. Il est mobilisé pour faire la jonction entre différentes activités. "II met de l'huile dans les rouages entre collègues et en famille ". Le service offre la possibilité de se partager entre différentes occupations, de moduler sa présence et son absence, de se mettre en retrait ou de s'intégrer, au gré des envies. L'engagement de l'acteur au sens d'audience, de disponibilité et d'attention qu'il prêtera sera variable car le service peut alors entrer en concurrence avec d'autres activités. II remplace « le tricot qu'on fait en regardant la télé ou en discutant en famille au salon qui permet d'être ouvert à la discussion ou de se fermer sur son ouvrage lorsqu'on veut la paix ».

L'usage devient un élément central qui facilite et catalyse les relations interpersonnelles : il permet de concilier l'individuel et le collectif. Les usagers le comparent souvent à un doudou ou à un coffre à jouets qui leur permet de ne jamais se sentir seul et abandonné, de ne plus s'ennuyer. Ils ont ainsi à leur disposition quelqu'un à appeler ou une pratique ludique ou culturelle à laquelle accéder (musique, jeu, film...) pour se divertir ou s'isoler. En cela, les applications de communication et les applications multimédia du mobile leurs paraissent complémentaires. En effet, elles permettent d'être seul lorsqu'ils en ont envie ou d'entrer en contact avec un tiers distant (appel, SMS) ou coprésent (en regardant ensemble). Le mobile serait à la fois pont, porte, fenêtre et route au sens de Simmel (1957), comme variable d'ajustement d'une nouvelle présence au monde.

Par ailleurs, la TV mobile abolit deux limites, l'une spatiale, l'autre temporelle. La première, liée à la mobilité n'est pas une attente en soi puisque plus de deux tiers des usages se font en situation postée. Le tiers qui reste suit une mobilité relative car liée à un déplacement global (se rendre au bureau de Poste chercher un colis). Les usagers se cherchent des niches protégées pour se poser, ou à défaut ils renoncent à l'usage. En reprenant la différence de Podnar 
Dans quelle mesure la TV sur mobile est-elle interactive?

et al. (2002), cela s'apparente davantage au nomadisme qu'à la mobilité : l'usager nomade se connecte au réseau de n'importe quel endroit et il en change régulièrement, alors que l'usager mobile utilise le service en se déplaçant. La liberté temporelle en revanche fait l'objet d'une véritable attente relative à une délinéarisation de la TV mobile pour s'affranchir ainsi de la grille horaire. Pourtant la délinéarisation attendue n'est pas totale mais limitée à une plage temporelle de quinze jours. En effet, les usagers ne souhaitent pas accéder à la série Colombo si celle-ci ne passe pas au même moment. La télévision ayant un usage relationnel, ils aiment pouvoir en parler autour d'eux et si personne n'a vu l'émission la veille ou les jours précédents, cela perd de son intérêt. II s'agit plutôt de remplacer le magnétoscope pour " rattraper » ce qui passe et qu'on ne peut pas voir pour des raisons extérieures. La série Colombo redeviendra intéressante dès lors qu'elle passera sur une chaîne.

En matière de rôle, les usagers déclarent ne pas souhaiter en explorer d'autre que celui de spectateur. En revanche, ils attendent un véritable outil de navigation qui permette selon l'envie soit de retrouver rapidement l'émission qu'ils cherchent ou qui peut leur plaire, soit de voir les nouveautés. A l'image des systèmes de navigation très sophistiqués qu'ils connaissent d'Internet, de tels systèmes reposeraient sur un profil de goûts à remplir en début de visionnage. De plus, ils s'adapteraient automatiquement en fonction des préférences de l'usager. Peu importe qu'il émane du fournisseur de contenu ou de l'opérateur. L'important est qu'on leur fasse des suggestions en fonction de ce qu'ils ont aimé, c'est-à-dire de faire évoluer dynamiquement le contenu en fonction des choix antérieurs. L'usage " lean back » plutôt passif serait privilégié puisque la connaissance des préférences de l'usager pourrait se faire en proposant une liste de contenus et en demandant de noter par ordre de préférence ce qu'ils aiment ou pas. Ceci permettrait de réaliser des playlist avec les programmes appréciés et dans un second temps de les partager avec ses amis ou avec des followers.

L'autre demande concerne l'interconnexion de la TV mobile avec les réseaux sociaux (Facebook, Twitter) afin de se voir proposer des contenus appréciés par ses amis ou de leur en suggérer. La pratique du tagging pourrait également se développer. Les répondants décrivent des usages en mobilité car ils souhaiteraient pouvoir à la fois recevoir les programmes vus par leurs amis et leur en envoyer immédiatement. Avertis directement sur leur profil Facebook, ces amis pourraient répondre, par un message sur Facebook, en appelant ou en envoyant un SMS. On passerait d'un usage solo à un usage collectif de la TV mobile. La TV mobile devient ainsi réellement innovante car elle permettrait non seulement de s'affranchir de l'espace et du temps, mais elle donnerait 
également aux usagers la possibilité d'émettre des contenus ou des nœuds de réseau qui réachemineraient ces contenus.

\section{Références bibliographie}

CHAMBAT P.,1994, « Usages des technologies de l'information et de la communication », Technologies et société, vol. 6, n³, pp. 249-270.

CHRISTENSEN, L. H., 2002, The impact of "interactivity" on television consumption - Stay tuned, visit our website, press the red button, working paper Dublin City University, <http://www.stem.dcu.ie/pdf/impact_of_interactivity.pdf>, dernièrement consulté le 19.02.2010.

COMMISSION GENERALE DE TERMINOLOGIE ET DE NEOLOGIE, 2009, Vocabulaire des techniques de l'information et de la communication, Paris, Commission Générale de Terminologie et de Néologie.

DONNAT O. (dir.), 2009, Les pratiques culturelles des Français à l'ère numérique, Paris, La Documentation française.

DOWNES E. J., S. J. MC MILAN, 2000, Defining interactivity : a qualitative identification of key dimensions, New Media \& Society, vol 2, pp 157-179.

FELDMAN T., 1997, Introduction to digital media, Londres, Routledge.

FLICHY P., 2001, L'imaginaire d'internet, Paris, La Découverte.

GARABUAU-MOUSSAOUI I., D. DESJEUX, 2000, Objet banal, objet social. Les objets quotidiens comme révélateurs des relations sociales, Paris, L'Harmattan.

GOGGIN, 2007, Cell phone culture, London, UK, Routledge.

HODGE W., 1994, Interactive television : a comprehensive guide for multimedia technologists, New York, Mac Grow Hill.

JENSEN J.F., 1999, The concept of interactivity, in Jensen J.F, Toscan C., Interactive television, Aalborg University Press, Aalborg

MEMMI A., 1979, La dépendance. Esquisse pour un portrait du dépendant, Paris, Gallimard.

O'HARA K., A. S. MITCHELL, A. VORBAU, 2007, " Consuming Video on Mobile Devices ", Proceedings of CHI '07, April 28 - May 3, 2007, San Jose, California, USA. 
Dans quelle mesure la TV sur mobile est-elle interactive?

PRONOVOST G., 1994, « Medias : éléments pour l'étude de a formation des usages ", Technologies de l'information et société, vol. 6, n4, pp. 377-400.

PODNAR I., M. HAUSWIRTH, M. JAZAYERE, 2002, « Mobile push: Delivering Content to Mobile Users ", Proceeding of the International Workshop on Distributed Event-Based Systems, Vienne, Autriche, pp. 563-570 ; http://citeseer.ist.psu.edu/508260.html, dernièrement consulté le 19.02.2010.

RUTTEN P., MANSHANDEN W., MUSKENS J., KOOPS O., 2003, De creative industrie in Amsterdam en de regio, TNO report SB 04-29-2004

SIMMEL G., 1957, Brücke und Tür, Stuttgart, Koehler.

SIMMEL G., 1908, La parure et autres essais, Paris, Maison des sciences de l'homme.

SINGLY, F. DE, 2001, Libres, ensemble, Paris, Odile Jacob.

SÖDERGARD C. (dir.), 2003, Mobile television - technology and user experiences. Report on the Mobile-TV project, VTT Information Technology, http://WWW.vtt.fi/inf/pdf/publications/2003/P506.pdf

STEUER J., 1995, « Defining virtual reality : dimensions determining telepresence ", dans F. BIOCCA, M. LEVY (dir.) Communication in the age of virtual reality, Hillsdale, NJ, pp 33-56.

VAN DEN BROECK W., B. LIEVENS, J. PIERSON, 2006, Domestication research for media and technology development: a case study, Conference Proceedings of IAMCR 2006, Le Caire, Egypte, 23-28 Juillet.

VAN DIJK J., A. HEUVELMAN, O. PETERS, 2003, Interactive television or enhanced television? The Dutch users interest in applications on ITV via setup boxes, paper presented at the annual conference of the International Communication Association in San Diego, USA, http://www.gw.utwente.nl/vandijk/itv/itv_plaatje/a-interactive-orenhanced_tv_i.pdf

VEBLEN T., 1970, La théorie de la classe du loisir, Paris, Gallimard. 